\title{
Optical coherence tomography features of retinal lesions in Chinese patients with endogenous Candida endophthalmitis
}

\author{
Hong Zhuang ${ }^{1,2+}$, Xinyi Ding ${ }^{1,2+}$, Fengjuan Gao ${ }^{1,2}$, Ting Zhang ${ }^{1,2}$, Yingqin Ni ${ }^{1,2}$, Qing Chang ${ }^{1,2}$ and Gezhi Xu ${ }^{1,2^{*}}$ (D)
}

\begin{abstract}
Background: To evaluate the optical coherence tomography (OCT) features of retinal lesions in Chinese patients with endogenous Candida endophthalmitis (ECE).

Methods: We performed a retrospective review of patients diagnosed with ECE at one medical center. The medical records of the patients including predisposing risk factors, treatment and visual acuity were reviewed. And we focused on the analysis of OCT images of retinal lesions before and after treatment.

Results: A total of 16 Chinese patients (22 eyes) were included in this study. The most frequent predisposing risk factors were intravenous use of corticosteroids or antibiotics, lithotripsy for urinary calculi, and diabetes. After treatment, visual acuity was improved in 13 (59.1\%) of the 22 eyes, and remained the same in the other 9 (40.9\%) eyes. Pre-treatment OCT images obtained at presentation were available for 17 of the 22 eyes. Four types of the OCT manifestations of retinal lesions were identified: type 1 (subretinal macular lesions), type 2 (lesions are located in the inner retinal layer), type 3 (lesions involve the full-thickness retina and accompanied with macular edema), type 4 (sub-inner limiting membrane lesions). Pre-treatment OCT imaging of the 17 eyes revealed five as type 1, four as type 2, six as type 3, and two as type 4. After treatment, OCT images revealed epiretinal membrane and subretinal fibrosis as the most common post-treatment complications of ECE. Epiretinal membrane was detected in 2/4 type 2 lesions, in 4/6 type 3 lesions, and in 1/2 type 4 lesions, while subretinal fibrosis was mainly seen in type 1 lesions (4/5). Among the types, visual prognosis was best in eyes with type 2 lesions.

Conclusions: In this case series, the OCT manifestations of retinal lesions in ECE could be classified into four types. The post-treatment OCT manifestations were different in four types of lesions. We preliminarily found that the OCT morphology of retinal lesions was associated with the visual prognosis of ECE.
\end{abstract}

Keywords: Endogenous endophthalmitis, Candida albicans, Retinal lesion, Optical coherence tomography

\section{Background}

Endogenous endophthalmitis, also called metastatic endophthalmitis, results from hematogenous spread of infectious microbes from distant foci. Endogenous endophthalmitis is a vision-threatening intraocular infection that accounts for approximately $2-10 \%$ of all cases of endophthalmitis [1-3], which is reportedly increasing in incidence in East Asian

\footnotetext{
*Correspondence: drxugezhi@163.com

${ }^{+}$Hong Zhuang and Xinyi Ding contributed equally to this work.

'Department of Ophthalmology, Eye and ENT Hospital of Fudan University, Shanghai 200031, China

${ }^{2}$ Key Laboratory of Visual Impairment and Restoration of Shanghai and Key Laboratory of Myopia of State Health Ministry, Fudan University, Shanghai 200031, China
}

population [4, 5]. The majority of cases of endogenous endophthalmitis are caused by fungal infections [6-8]. Endogenous fungal endophthalmitis is associated with underlying systemic risk factors that include recent major surgery, malignant tumor, systemic antibiotic use, diabetes mellitus, urinary tract infection, and indwelling catheter [9-11]. The majority of these cases are associated with Candida species, especially Candida albicans [12-15].

Candida species typically cause inflammation in the choroid, retina, and vitreous. The ophthalmoscopic appearance of endogenous Candida endophthalmitis (ECE) is characterized by one or more white lesions located mainly in the posterior pole of the fundus [16]. Further 
aggravation of ECE results in vitreous inflammation and disseminated lesions.

Most previous studies have focused on the clinical manifestations of ECE. Several single case studies have reported the manifestations of ECE on optical coherence tomography (OCT) [17-19]. A recent study has analyzed the OCT features of ECE in nine patients from four medical centers in Italy and Australia [20]. Although the incidence of ECE was increased in East Asia, there was still few OCT analysis of ECE in East Asian population.

Therefore, we retrospectively reviewed a case series of Chinese patients with ECE, focusing on the OCT features of ECE before and after treatment. We used OCT for classification and clinical evaluation of retinal lesions caused by Candida infection in patients with ECE.

\section{Methods}

We performed a retrospective review of patients diagnosed with ECE who visited the Eye and ENT Hospital of Fudan University, China, between January 2014 and April 2018. The research followed the tenets of the Declaration of Helsinki and was approved by the Ethics Committee of the Eye and ENT Hospital of Fudan
University. Written informed consent was obtained from each patient after explanation of the nature and possible consequences of the study.

ECE was diagnosed according to the patient's general condition, ocular manifestations, and microbial culture results. Patients with positive C. albicans cultures were included. The exclusion criteria were penetrating ocular trauma, recent intraocular surgery, or any other cause of intraocular inflammation. The medical records of the patients were reviewed to obtain the patients' age, sex, predisposing risk factors, treatment, and visual acuity; and their spectraldomain OCT images were analyzed in detail.

Patients received intraocular therapy and systemic antifungal therapy. Intraocular therapy included intravitreal injection of amphotericin B $(5 \mu \mathrm{g} / 0.1 \mathrm{ml})$ and/or vitrectomy. Patients who underwent vitrectomy routinely received an intravitreal injection of amphotericin B at the end of the surgery. All patients received systemic antifungal therapy for at least 2 months. The commonly used daily dose of oral antifungal drugs (fluconazole, itraconazole, or voriconazole) was $400 \mathrm{mg}$. All patients underwent comprehensive ophthalmic examinations, including slit-lamp biomicroscopy, indirect ophthalmoscopy, measurement of best-corrected

Table 1 Clinical characteristics of 22 eyes with ECE

\begin{tabular}{|c|c|c|c|c|c|c|c|}
\hline Patient No. & Age/sex & Eye & Predisposing risk factors & C. a culture & Treatment & Initial VA & $\overline{\text { Last VA }}$ \\
\hline 1 & $41 / M$ & $\mathrm{OD}$ & Intravenous corticosteroid & Vitreous (+) & $\mathrm{IVI}+$ vitrectomy & $20 / 2000$ & $20 / 400$ \\
\hline 2 & 29/F & OS & Intravenous antibiotics & Vitreous (+) & $\mathrm{IVI}+$ vitrectomy & $20 / 2000$ & $20 / 2000$ \\
\hline 3 & $28 / M$ & OS & Endocarditis & blood $(+)$ & $\mathrm{IVI}+$ vitrectomy & $20 / 100$ & $20 / 100$ \\
\hline 4 & $40 / F$ & OS & Intravenous corticosteroid & Vitreous (+) & $\mathrm{IVI}+$ vitrectomy & $\mathrm{HM}$ & $\mathrm{HM}$ \\
\hline \multirow[t]{2}{*}{5} & $58 / \mathrm{M}$ & $\mathrm{OD}$ & Intravenous corticosteroid & Vitreous (+) & $|\mathrm{V}|$ & $20 / 200$ & $20 / 63$ \\
\hline & & OS & & Vitreous (+) & $|\mathrm{V}|$ & $20 / 200$ & $20 / 100$ \\
\hline 6 & $23 / F$ & OS & Diabetes & Vitreous (+) & $\mathrm{IVI}+$ vitrectomy & $\mathrm{HM}$ & $20 / 100$ \\
\hline 7 & $67 / F$ & OS & Intravenous corticosteroid & Vitreous (+) & $\mathrm{IVI}+$ vitrectomy & CF & CF \\
\hline \multirow[t]{2}{*}{8} & $49 / F$ & $\mathrm{OD}$ & nephrolithotripsy & blood $(+)$ & $|\mathrm{V}|$ & $20 / 400$ & $20 / 100$ \\
\hline & & OS & & & $|\mathrm{V}|$ & $20 / 32$ & $20 / 25$ \\
\hline \multirow[t]{2}{*}{9} & $58 / F$ & OD & Diabetes & Vitreous (+) & $\mathrm{IVI}+$ vitrectomy & $20 / 2000$ & $20 / 200$ \\
\hline & & OS & & Vitreous (+) & $\mathrm{IVI}+$ vitrectomy & CF & CF \\
\hline \multirow[t]{2}{*}{10} & $37 / F$ & $\mathrm{OD}$ & Diabetes; nephritis & blood $(+)$; midstream urine $(+)$ & $|\mathrm{V}|$ & $20 / 25$ & $20 / 20$ \\
\hline & & OS & & & $\mathrm{IVI}+$ vitrectomy & LP & LP \\
\hline \multirow[t]{2}{*}{11} & $51 / F$ & OD & Intravenous antibiotics & Vitreous (+) & $\mathrm{IVI}+$ vitrectomy & CF & $20 / 400$ \\
\hline & & OS & & Vitreous (+) & $\mathrm{IVI}+$ vitrectomy & CF & CF \\
\hline 12 & $53 / F$ & OS & ureterolithotripsy & blood $(+)$ & $|\mathrm{V}|$ & $20 / 63$ & $20 / 50$ \\
\hline 13 & $39 / M$ & $\mathrm{OD}$ & ureterolithotripsy & Vitreous (+) & $\mathrm{IVI}+$ vitrectomy & $20 / 1000$ & $20 / 1000$ \\
\hline \multirow[t]{2}{*}{14} & $68 / M$ & OD & nephrolithotripsy & blood $(+)$; midstream urine $(+)$ & $|\mathrm{V}|$ & $20 / 100$ & $20 / 63$ \\
\hline & & OS & & & $\mathrm{IVI}+$ vitrectomy & CF & $20 / 160$ \\
\hline 15 & $59 / F$ & $\mathrm{OD}$ & Gastrointestinal surgery & blood $(+)$ & $|\mathrm{V}|$ & $\mathrm{HM}$ & $\mathrm{HM}$ \\
\hline 16 & $28 / \mathrm{F}$ & OD & Abortion & Vitreous (+) & $\mathrm{IVI}+$ vitrectomy & $\mathrm{HM}$ & CF \\
\hline
\end{tabular}

ECE Endogenous candida endophthalmitis, F. Female, M Male, OD Right eye, OS Left eye, C. a Candida albicans, IVI Intravitreal injection, VA Visual acuity, LP Light perception, HM Hand motion, CF Counting fingers 
visual acuity, B-scan ultrasonography, fundus photography, and spectral-domain OCT.

OCT images were obtained using the Spectralis spectral-domain OCT instrument (Heidelberg Engineering, Heidelberg, Germany). The most obvious retinal lesion in each eye was examined by OCT. After treatment, the eyes were re-scanned using the eye-tracking-based follow-up function in Spectralis OCT. Therefore, the same lesion site was examined, thereby facilitating precise evaluation of the post-treatment changes in the retinal lesions. If OCT images could not be obtained at presentation because of severe vitreous inflammation, the patient underwent OCT examination after vitrectomy.

When comparing the initial visual acuity (before treatment) and the last visual acuity (after treatment), the visual acuity value was converted to logarithm of minimal angle of resolution (LogMAR) unit. According to the literatures [21, 22], visual acuity of counting fingers or worse was converted to LogMAR unit as follows: counting fingers equal to $2.0 \log M A R$, hand motion equal to 2.3 LogMAR, light perception equal to 2.5 LogMAR. The paired Wilcoxon rank sum test was performed with Stata 11.0 statistical software (Stata Corporation, College Station, TX, USA). A $P$ value of $<0.05$ was considered statistically significant.

\section{Results}

\section{Demographic and clinical characteristics}

A total of 16 Chinese patients (22 eyes, 5 males and 11 females) were included in this retrospective observational study. Of these patients, 10 had unilateral ECE
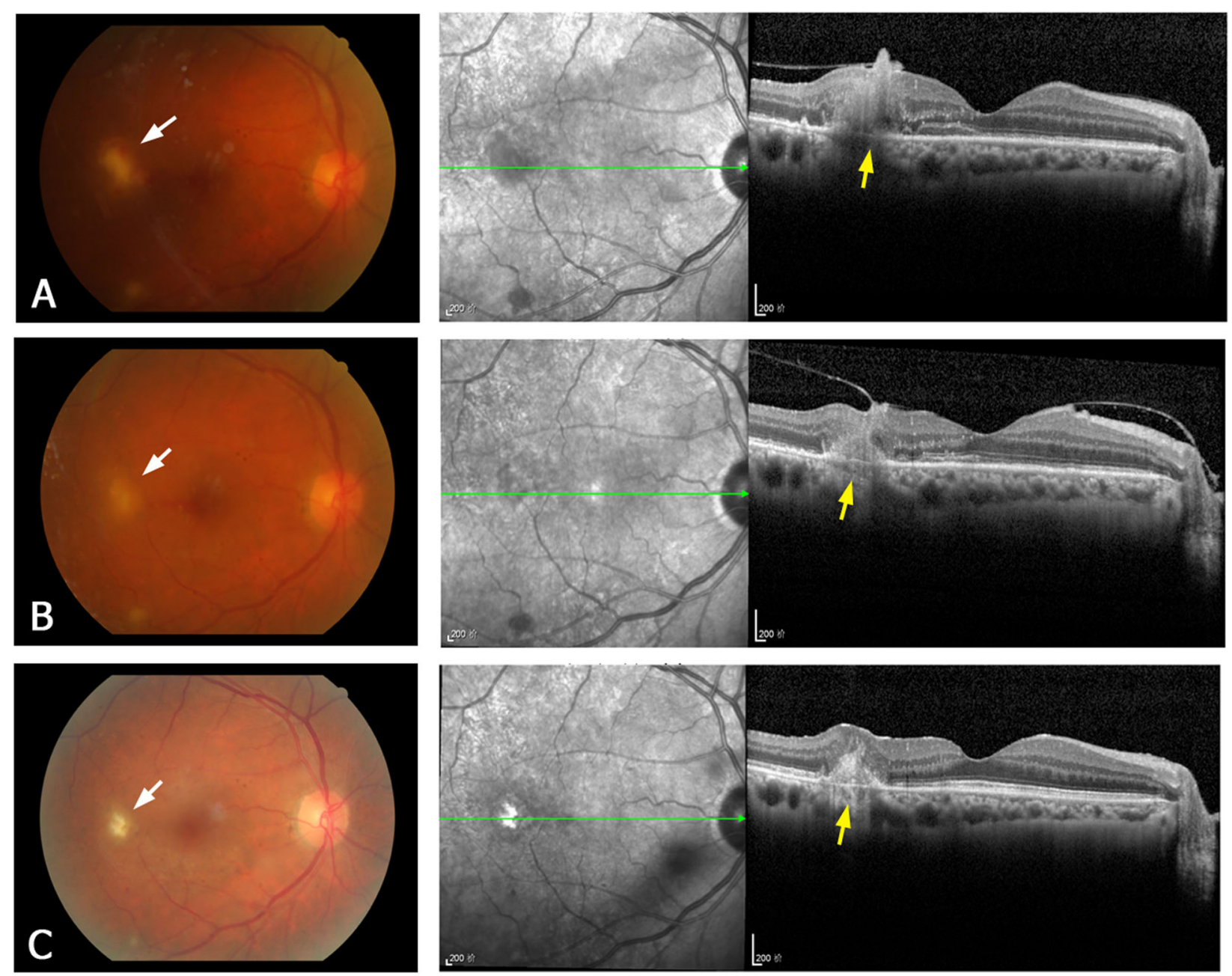

Fig. 1 A representative type 1 lesion of ECE. Fundus photographs (left column) and OCT images (right column) of the right eye of patient 5. a Before treatment. The fundus photograph shows a yellow-white lesion with a small hemorrhage (white arrow) on the temporal side of the fovea. The OCT image shows a macular subretinal lesion originating at the retinal pigment epithelium/choroid layer (yellow arrow) and penetrating into the neurosensory retina. $\mathbf{b}$ Two weeks after treatment (intravitreal injection). Shrinkage of the subretinal lesion is apparent on the OCT image. c Five months after treatment. The OCT image shows hyperreflective fibrosis in the subretinal lesion. The inner choroid is also hyperreflective 
and 6 had bilateral ECE. The mean age of the patients was $45.5 \pm 14.4$ years (range, 23-68 years).

The most frequent predisposing risk factors were intravenous use of corticosteroids or antibiotics (in six patients), lithotripsy of urinary calculi (in four), and diabetes (in three, diabetes combined with nephritis in one). All ECE diagnoses were confirmed by microbial cultures. Ten patients (13 eyes) had vitreous cultures positive for $C$. albicans, and the other six patients ( 9 eyes) had blood cultures positive for $C$. albicans (two of whom also had a positive midstream urine culture). The positive rate of vitreous fungal culture in vitrectomy samples $(78.6 \%$; 11/14) was higher than that in needle biopsy samples $(25.0 \%, 2 / 8)$.

Intraocular therapy included intravitreal injection of amphotericin B or vitrectomy; 8 eyes received intravitreal injection of amphotericin B only, and 14 eyes received pars plana vitrectomy. The mean follow-up duration was $7.3 \pm 4.1$ months (range, $2-18$ months). After intraocular therapy and systemic antifungal therapy, the endogenous endophthalmitis caused by Candida infection was effectively controlled. The visual acuity after treatment (mean LogMAR $1.25 \pm 0.78$ ) was significantly better than the visual acuity before treatment (mean LogMAR $1.59 \pm 0.75)(P=0.0015)$. The visual acuity was improved in 13 (59.1\%) eyes and remained the same in 9 $(40.9 \%)$ eyes. Eleven (50\%) eyes had a visual acuity of $20 /$ 200 or better at the last visit. The clinical characteristics of the 22 eyes with ECE are listed in Table 1.

\section{OCT findings}

Pre-treatment OCT was performed at presentation in 17 of the 22 eyes, but not in the other 5 eyes because of
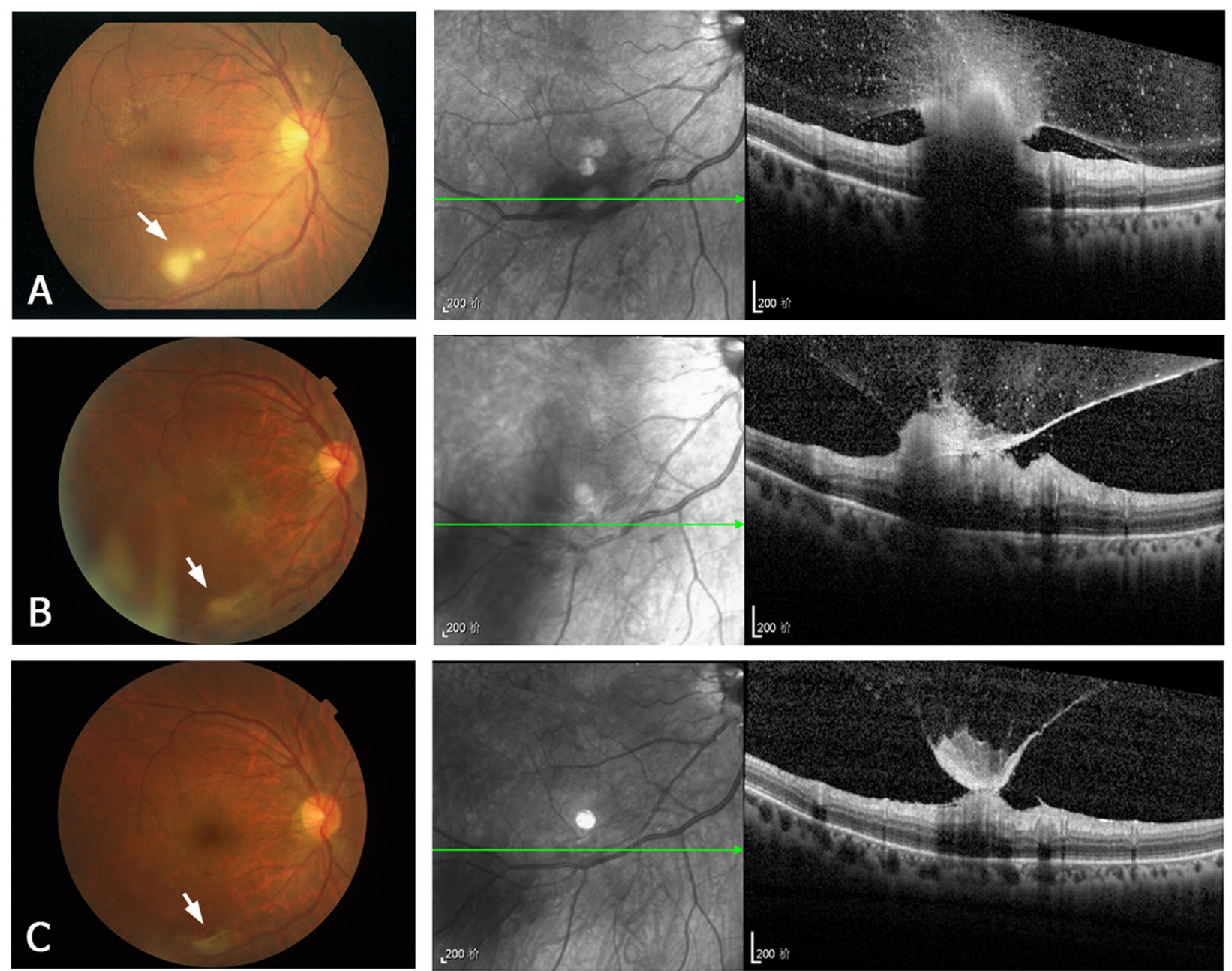

Fig. 2 A representative type 2 lesion of ECE. Fundus photographs (left column) and OCT images (right column) of the right eye of patient 10. a Before treatment. The fundus photograph shows a white round lesion (approximately one-half disc diameter) near the infratemporal vascular arcade (white arrow). The OCT image shows a lesion in the inner retinal layer, invading the posterior vitreous. No intra- or subretinal fluid is present. $\mathbf{b}$ One month after treatment (intravitreal injection). The OCT image shows shrinkage of the retinal lesion. $\mathbf{c}$ Three months after treatment. In the OCT image, regression of the retinal lesion and formation of epiretinal membrane are apparent 
severe vitreous inflammation. According to the retinal layer of lesion invasion and the lesion location, four types of the OCT manifestations of the retinal lesions were identified, as follows. Type $\mathbf{1}$ are subretinal macular lesions that originate from choroid and penetrate the neurosensory retina (Fig. 1a). Type 2 lesions are located in the inner retinal layer and protrude into the vitreous, without intra- or subretinal fluid (Fig. 2a). They are always located close to the retinal vascular arcades and are relatively uniform in size, ranging from one-fourth to one disc diameter. Type 3 lesions involve the fullthickness retina and protrude into the vitreous (Fig. 3a). These lesions always cause macular edema. Type 4 lesions are located in the sub-inner limiting membrane
(ILM) of the macula, and break through the ILM into the vitreous (Fig. 4a). Type 4 lesions exceed 2 disc diameters.

Pre-treatment OCT imaging of the 17 eyes revealed five as type 1 , four as type 2 , six as type 3 , and two as type 4 (Table 2). Table 2 also lists the post-treatment OCT features in the 22 eyes. OCT imaging after treatment revealed regression of the retinal lesion and resolution of the macular edema, and identified epiretinal membrane and subretinal fibrosis as the most common complications of ECE. After treatment, the epiretinal membrane was detected in 2/4 type 2 lesions, in 4/6 type 3 lesions, and in $1 / 2$ type 4 lesions. Among the four types, the subretinal fibrosis mainly existed in type 1
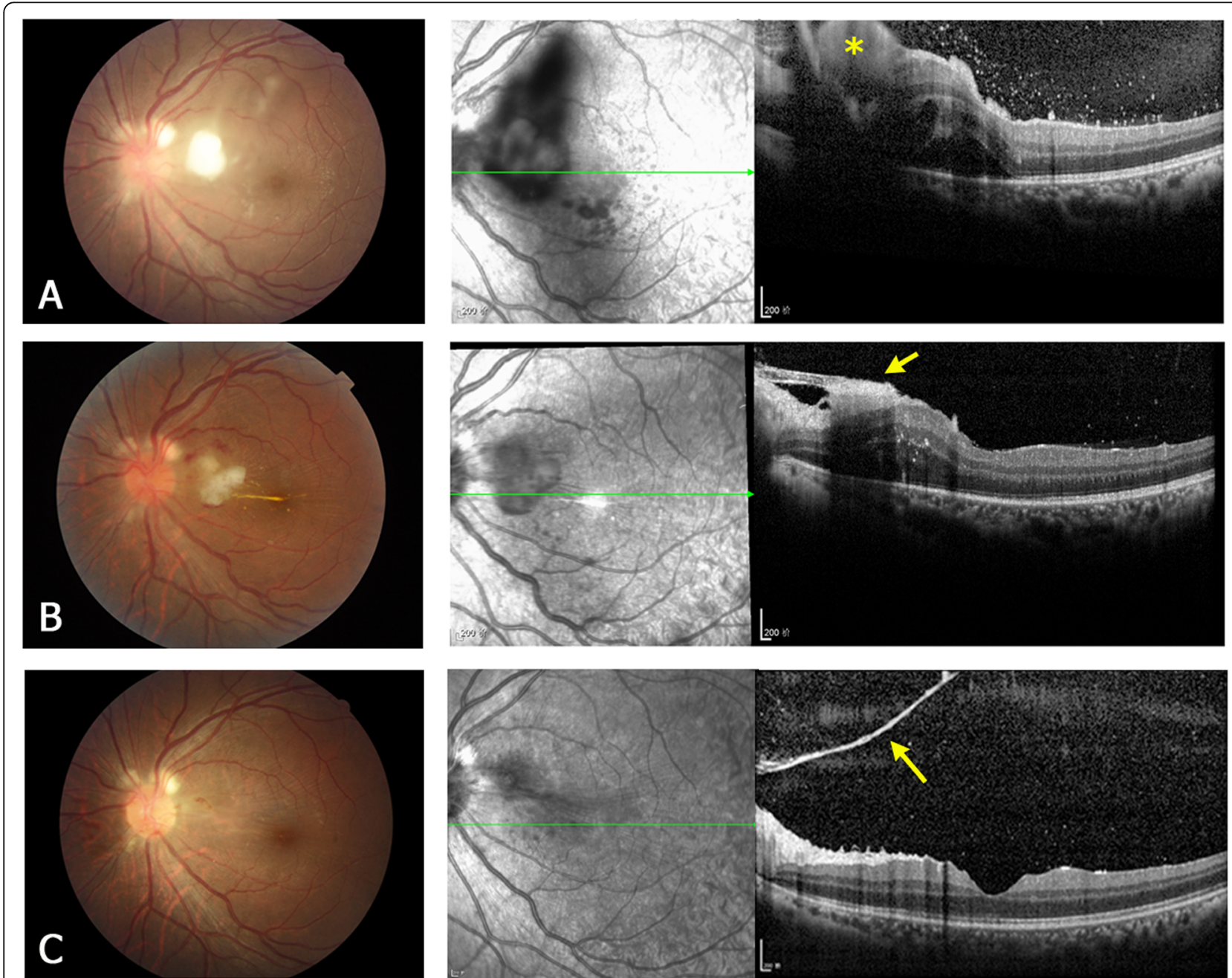

Fig. 3 A representative type 3 lesion of ECE. Fundus photographs (left column) and OCT images (right column) of the left eye of patient 3. a Before treatment. The fundus photograph shows a white fluffy lesion at the posterior pole. The OCT image shows a highly reflective lesion (yellow asterisk) involving the full-thickness retina and protruding into the vitreous. Macular edema is present with subretinal fluid. The hyperreflective dots in the posterior vitreous are infiltrating inflammatory cells. $\mathbf{b}$ One week after treatment (vitrectomy). The OCT image shows shrinkage of the retinal lesion, formation of epiretinal membrane (yellow arrow) and reduction of the macular edema. c Three months after treatment. The retinal lesion almost resolves, and OCT shows a residual pre-retinal membrane (yellow arrow) close to the optic disc 

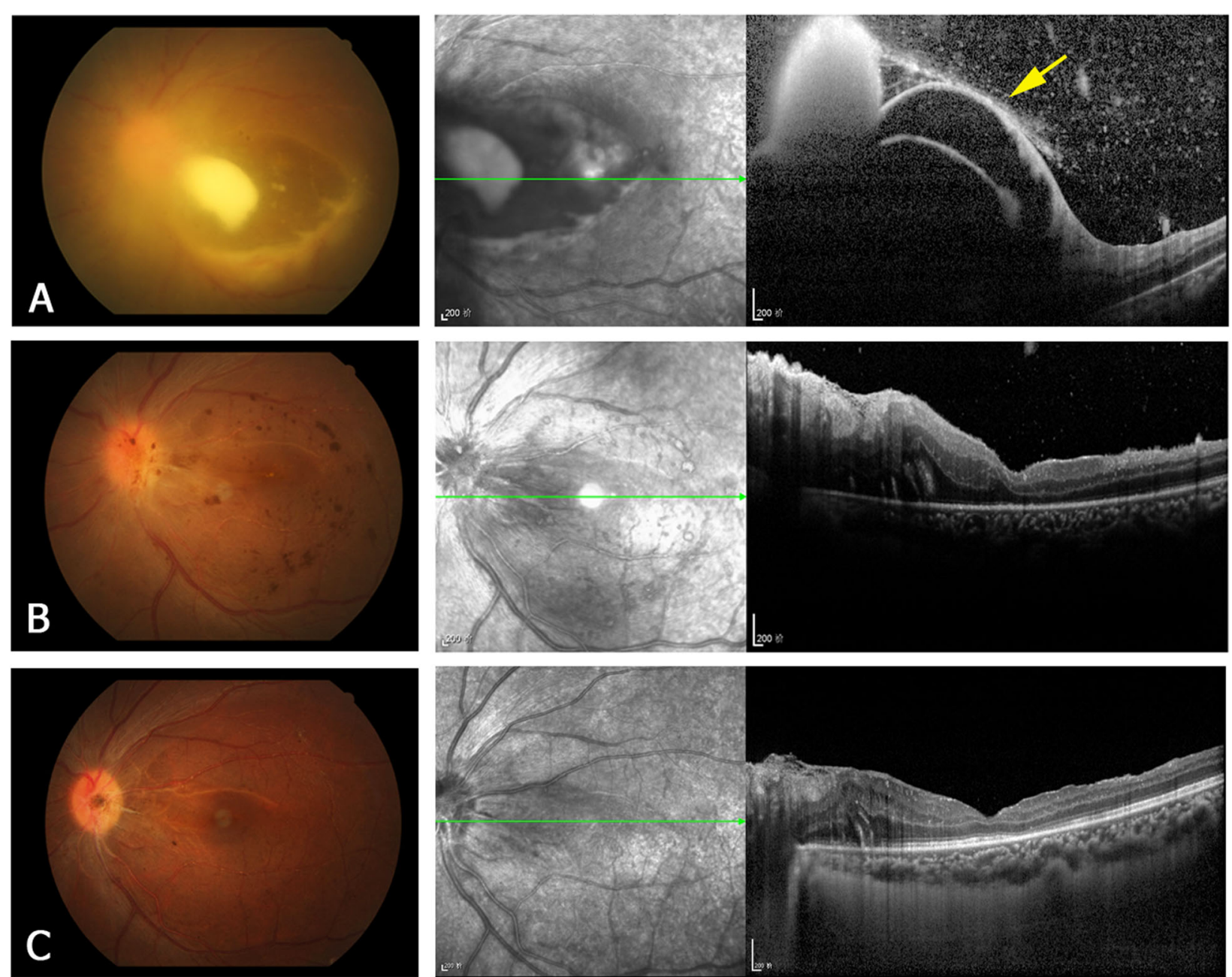

Fig. 4 A representative type 4 lesion of ECE. Fundus photographs (left column) and OCT images (right column) of the left eye of patient 6 . a Before treatment. The fundus photograph shows a large lesion at the posterior pole. The OCT image shows detachment of the ILM (yellow arrow) and the sub-ILM lesion breaking through the ILM into the vitreous. $\mathbf{b}$ Two weeks after treatment (vitrectomy). In the OCT image, the retinal lesion is no longer apparent, but the nasal retinal edema remains. c Three months after treatment. The OCT image shows reduction of the retinal edema and improvement of the macular foveal structure

lesions (4/5). The representative OCT follow-up images of each lesion type are shown in Figs. 1, 2, 3 and 4. Table 3 lists the visual prognoses of the different types of retinal lesions. Among the four types, eyes with type 2 lesions had the best baseline and final visual acuities.

\section{Discussion}

ECE is associated with numerous underlying systemic risk factors. In the present patients, the major risk factors for ECE were intravenous use of corticosteroids or antibiotics, lithotripsy of urinary calculi, and diabetes, all of which have been reported previously [9-11]. Other risk factors were endocarditis, gastrointestinal surgery, and abortion [23].

Of the 22 eyes in our study, 14 were treated with vitrectomy. The vitreous opacity and pathogens in the vitreous cavity can be removed by vitrectomy. Vitrectomy is necessary for cases of severe vitreous inflammation and disseminated lesions in the vitreous. If intravitreal injection of amphotericin B cannot control the endophthalmitis, vitrectomy can be performed. It has been reported that the rate of a positive vitreous culture was higher with vitrectomy than with vitreous needle biopsy $[8,24]$. Our study confirmed that the positive rate of vitreous fungal culture in vitrectomy samples $(78.6 \%)$ was higher than that in needle biopsy samples $(25.0 \%)$.

Several case reports have described the OCT findings of subretinal ECE lesions [17-19]. A recent study [20] analyzed the OCT features of ECE in nine patients from Italy and Australia, and described two OCT patterns of retinal ECE lesions: intraretinal (the 
Table 2 OCT features of 22 eyes with ECE

\begin{tabular}{|c|c|c|c|}
\hline Case & Eye & OCT pre-treatment & OCT post-treatment \\
\hline 1 & OD & Type 3 & lesion regressed, ME resolved \\
\hline 2 & OS & Type 1 & lesion shrunk, fibrosis of lesion \\
\hline 3 & OS & Type 3 & lesion regressed, ME resolved, ERM \\
\hline 4 & OS & NA & lesion regressed, subretinal fibrosis, ERM \\
\hline \multirow[t]{2}{*}{5} & OD & Type 1 & lesion shrunk, fibrosis of lesion \\
\hline & OS & Type 1 & lesion regressed, RPE alteration \\
\hline 6 & OS & Type 4 & lesion regressed \\
\hline 7 & OS & NA & lesion regressed, subretinal fibrosis, ERM \\
\hline \multirow[t]{2}{*}{8} & OD & Type 3 & lesion regressed, subretinal fibrosis, ERM \\
\hline & OS & Type 2 & lesion regressed \\
\hline \multirow[t]{2}{*}{9} & OD & Type 1 & lesion shrunk, fibrosis of lesion \\
\hline & OS & Type 1 & lesion shrunk, fibrosis of lesion \\
\hline \multirow[t]{2}{*}{10} & OD & Type 2 & lesion regressed, ERM \\
\hline & OS & Type 4 & lesion regressed, severe ERM \\
\hline \multirow[t]{2}{*}{11} & OD & NA & subretinal fibrosis \\
\hline & OS & NA & subretinal fibrosis, ERM \\
\hline 12 & OS & Type 2 & lesion regressed, ERM \\
\hline 13 & $\mathrm{OD}$ & Type 3 & lesion regressed, ME reduced \\
\hline \multirow[t]{2}{*}{14} & OD & Type 2 & lesion regressed \\
\hline & OS & NA & lesion regressed, ERM \\
\hline 15 & OD & Type 3 & lesion regressed, severe ERM \\
\hline 16 & OD & Type 3 & lesion regressed, ERM \\
\hline
\end{tabular}

OCT Optical coherence tomography, ECE Endogenous candida endophthalmitis, $O D$ Right eye, $O S$ Left eye, NA Non-available (cannot obtain pre-treatment OCT images), ERM Epiretinal membrane, ME Macular edema, RPE Retinal pigment epithelium

lesion is confined to the inner retinal layer or extends into the vitreous) and chorioretinal (the subretinal lesion penetrates through the retina into the vitreous) patterns. In our study involving Chinese patients, four OCT types of retinal ECE lesions were identified. Types 1 (subretinal lesion in the macula) and 2 (lesion in the inner retinal layer) are respectively similar to the chorioretinal and intraretinal patterns described before [20]. Type 3 lesions involve the full thickness of the retina, and cause massive inflammatory cells in the posterior vitreous. Type 3 lesions are always accompanied with macular edema and subretinal fluid, but without obvious subretinal infection lesions. Type 4 lesions are relatively rare but special. The growth of Candida lesions in the retina results in detachment of the ILM. To our knowledge, this is the first report of type 4 lesions in ECE.

There have been very few OCT follow-up studies of ECE after treatment. Even in the abovementioned OCT study [20] of patients with ECE, follow-up images were not available for most patients. In our study, all 22 eyes were followed-up by OCT for at least 2 months at the same medical center, and posttreatment changes in the retinal lesions were evaluated using the eye-tracking-based follow-up function of the OCT instrument. These images revealed the most common complications of ECE after treatment were epiretinal membrane and subretinal fibrosis. We found that the post-treatment OCT manifestations were different in four types of lesions. Epiretinal membrane was detected in type 2 lesions, type 3 lesions, and type 4 lesions, while subretinal fibrosis was mainly seen in type 1 lesions.

As reported previously [25], ECE is associated with a high rate of vision loss. Factors associated with poor visual outcomes include poor baseline visual acuity and centrally located fungal lesions. Our analysis of visual prognosis among the four retinal lesion types revealed that the eyes with type 2 lesions had better baseline visual acuity. After antifungal therapy without vitrectomy, the final visual acuity of all eyes with type 2 lesions was improved. This may be explained by the distance between type 2 lesions and the macular fovea.

Due to the limited number of cases included, this study could not provide a detailed statistical analysis of visual prognosis of different retinal lesions. Further larger sample size study is needed to confirm our OCT classification of retinal lesions and related visual prognosis.

\section{Conclusion}

Compared with the previous reports, this study has broadened our understanding of the OCT features of ECE. In this case series, we preliminarily found that the OCT morphology of retinal lesions was associated with the visual prognosis of ECE. And OCT can be used to monitor the therapeutic effects on ECE.

Table 3 Visual prognosis of different types of retinal lesions in eyes with ECE

\begin{tabular}{llllll}
\hline & NA & Type 1 & Type 2 & Type 3 & Type 4 \\
\hline Initial VA $(>=20 / 200)$ & $0 / 5(0 \%)$ & $2 / 5(40 \%)$ & $4 / 4(100 \%)$ & $1 / 6(16.7 \%)$ & $3 / 2(0 \%)$ \\
Last VA $(>=20 / 200)$ & $1 / 5(20 \%)$ & $3 / 5(60 \%)$ & $4 / 4(100 \%)$ & $3 / 6(50 \%)$ & $1 / 2(50 \%)$ \\
VA improved & $2 / 5(40 \%)$ & $3 / 5(60 \%)$ & $4 / 4(100 \%)$ & $3 / 6(50 \%)$ & $1 / 2(50 \%)$ \\
\hline
\end{tabular}

VA Visual acuity, NA Non-available (cannot obtain pre-treatment OCT images) 


\section{Supplementary information}

Supplementary information accompanies this paper at https://doi.org/10. 1186/s12886-020-01337-9.

Additional file 1. Supplemental Table 1. Symptom duration before treatment in 22 eyes.

\section{Abbreviations}

C. a: Candida albicans; ECE: Endogenous Candida endophthalmitis; ERM: Epiretinal membrane; ILM: Inner limiting membrane; ME: Macular edema; OCT: Optical coherence tomography; RPE: Retinal pigment epithelium; VA: Visual acuity

\section{Acknowledgements}

Not applicable.

\section{Authors' contributions}

$\mathrm{HZ}$ and $\mathrm{XD}$ conducted the research and drafted the manuscript. FG, TZ and YN analyzed and interpreted the data. QC and GX conceived and designed the study, and also made critical revision to the manuscript. All authors read and approved the final manuscript.

\section{Funding}

This work was supported by the grant from the Shanghai Hospital Development Center (SHDC12016116), and National Natural Science Foundation of China (Grant no. 81600739 and 81570855). The funders had no role in the design of the study, interpretation of data or writing the manuscript.

\section{Availability of data and materials}

The data used in the current study is available from the corresponding author upon request.

\section{Ethics approval and consent to participate}

The present study was conducted in accordance with the principles of the Declaration of Helsinki and was approved by the Ethics Committee of the Eye and ENT Hospital of Fudan University. Written informed consent was obtained from each patient.

\section{Consent for publication}

Written informed consents were obtained from the patients for publication of any information contained within the manuscript. A copy of the written consent is available for review by the Editor of this journal.

\section{Competing interests}

The authors declare that they have no competing interests.

Received: 27 October 2019 Accepted: 7 February 2020

Published online: 14 February 2020

\section{References}

1. Okada AA, Johnson RP, Liles WC, D'Amico DJ, Baker AS. Endogenous bacterial endophthalmitis. Report of a ten-year retrospective study. Ophthalmology. 1994;101(5):832-8.

2. Shrader SK, Band JD, Lauter CB, Murphy P. The clinical spectrum of endophthalmitis: incidence, predisposing factors, and features influencing outcome. J Infect Dis. 1990;162(1):115-20

3. Ramakrishnan R, Bharathi MJ, Shivkumar C, Mittal S, Meenakshi R, Khadeer MA, et al. Microbiological profile of culture-proven cases of exogenous and endogenous endophthalmitis: a 10-year retrospective study. Eye (Lond). 2009;23(4):945-56

4. Wong JS, Chan TK, Lee HM, Chee SP. Endogenous bacterial endophthalmitis: an east Asian experience and a reappraisal of a severe ocular affliction. Ophthalmology. 2000;107(8):1483-91.

5. Lim HW, Shin JW, Cho HY, Kim HK, Kang SW, Song SJ, et al. Endogenous endophthalmitis in the Korean population: a six-year retrospective study. Retina. 2014;34(3):592-602.

6. Duan F, Yang Y, Yuan Z, Zheng Y, Cheng Z, Lin X. Clinical features and visual acuity outcomes in culture-positive endogenous fungal endophthalmitis in southern China. J Ophthalmol. 2017;2017:3483497.
7. Schiedler V, Scott IU, Flynn HW Jr, Davis JL, Benz MS, Miller D. Cultureproven endogenous endophthalmitis: clinical features and visual acuity outcomes. Am J Ophthalmol. 2004;137(4):725-31.

8. Zhang YQ, Wang WJ. Treatment outcomes after pars plana vitrectomy for endogenous endophthalmitis. Retina. 2005;25(6):746-50.

9. Tanaka M, Kobayashi Y, Takebayashi H, Kiyokawa M, Qiu H. Analysis of predisposing clinical and laboratory findings for the development of endogenous fungal endophthalmitis. A retrospective 12-year study of 79 eyes of 46 patients. Retina. 2001;21(3):203-9.

10. Shen X, Xu G. Vitrectomy for endogenous fungal endophthalmitis. Ocul Immunol Inflamm. 2009:17(3):148-52.

11. Sadiq MA, Hassan M, Agarwal A, Sarwar S, Toufeeq S, Soliman MK, et al. Endogenous endophthalmitis: diagnosis, management, and prognosis. Ophthalmic Inflamm Infect. 2015;5(1):32.

12. Lingappan A, Wykoff CC, Albini TA, Miller D, Pathengay A, Davis JL, et al. Endogenous fungal endophthalmitis: causative organisms, management strategies, and visual acuity outcomes. Am J Ophthalmol. 2012:153(1):162-6.

13. Takebayashi H, Mizota A, Tanaka M. Relation between stage of endogenous fungal endophthalmitis and prognosis. Graefes Arch Clin Exp Ophthalmol. 2006:244(7):816-20.

14. Ueda T, Takesue Y, Tokimatsu I, Miyazaki T, Nakada-Motokawa N, Nagao M, et al. The incidence of endophthalmitis or macular involvement and the necessity of a routine ophthalmic examination in patients with candidemia. PLoS One. 2019;14(5):e0216956.

15. Ranjith K, Sontam B, Sharma S, Joseph J, Chathoth KN, Sama KC, et al. Candida species from eye infections: drug susceptibility, virulence factors, and molecular characterization. Invest Ophthalmol Vis Sci. 2017;58(10):42019.

16. Chignell AH. Endogenous candida endophthalmitis. J R Soc Med. 1992; 85(12):721-4.

17. Imago M, Imai H, Nakanishi $Y$, Azumi A. Optical coherence tomography for monitoring the process of Candida endophthalmitis. Acta Ophthalmol. 2009:87(6):680-2.

18. Cho M, Khanifar AA, Chan RV. Spectral-domain optical coherence tomography of endogenous fungal endophthalmitis. Retin Cases Brief Rep. 2011;5(2):136-40.

19. Adam MK, Rahimy E. Enhanced depth imaging optical coherence tomography of endogenous fungal chorioretinitis. JAMA Ophthalmol. 2015; 133(11):e151931.

20. Invernizzi A, Symes R, Miserocchi E, Cozzi M, Cereda M, Fogliato G, et al. Spectral domain optical coherence tomography findings in endogenous Candida endophthalmitis and their clinical relevance. Retina. 2018;38(5): 1011-8.

21. Lange C, Feltgen N, Junker B, Schulze-Bonsel K, Bach M. Resolving the clinical acuity categories "hand motion" and "counting fingers" using the Freiburg Visual Acuity Test (FrACT). Graefes Arch Clin Exp Ophthalmol. 2009; 247(1):137-42

22. Lu X, Xia H, Jin C, Chen W, Siu-Chun Ng D, Yan H, et al. Prognostic factors associated with visual outcome of salvageable eyes with posttraumatic endophthalmitis. Sci Rep. 2019:9(1):12678.

23. Chen SJ, Chung YM, Liu JH. Endogenous Candida endophthalmitis after induced abortion. Am J Ophthalmol. 1998;125(6):873-5.

24. William A, Spitzer MS, Deuter C, Blumenstock G, Partsch M, Voykov B, et al. Outcomes of primary transconjunctival 23-gauge vitrectomy in the diagnosis and treatment of presumed endogenous fungal endophthalmitis. Ocul Immunol Inflamm. 2017;25(2):239-45.

25. Sallam A, Taylor SR, Khan A, McCluskey P, Lynn WA, Manku K, et al. Factors determining visual outcome in endogenous Candida endophthalmitis. Retina. 2012:32(6):1129-34.

\section{Publisher's Note}

Springer Nature remains neutral with regard to jurisdictional claims in published maps and institutional affiliations. 